\title{
Achalasia and Down syndrome: a unique association not to be missed
}

Guadalupe Viegelmann ${ }^{1}$, MD, Yee $\underline{\text { Low }}^{2}$, FRCS, FAMS, Bhavani Sriram ${ }^{3}$, MBBS, MRCP, Hui Ping $\underline{\mathrm{Chu}}^{4}$, MBBS, MRCPCH

\begin{abstract}
Achalasia is a rare primary oesophageal motility disorder that presents as a functional obstruction at the oesophago-gastric junction. The prevalence of achalasia in Down syndrome is much higher, which implies a unique association between these two uncommon conditions. Although the exact aetiology of achalasia is unknown, studies have proposed that its pathogenesis is related to autoimmune, infectious or genetic factors, leading to the intrinsic loss of inhibitory myenteric neurons in both the oesophagus and lower oesophageal sphincter. We herein report the case of a 16-month-old girl with Down syndrome and achalasia who was initially treated for gastro-oesophageal reflux disease. The diagnosis of achalasia was made only when her condition deteriorated, with subsequent failure to thrive, and upon further investigations, including barium swallow study and upper endoscopy. We also review the various mechanisms postulated in the development of achalasia in Down syndrome, as well as the various treatment modalities available for this rare disorder.
\end{abstract}

Keywords: achalasia, Down syndrome, gastro-oesophageal reflux disease

\section{INTRODUCTION}

Achalasia is a rare motor disorder of the oesophagus presenting as a functional obstruction at the oesophago-gastric junction. The prevalence of achalasia in Down syndrome has been reported to exceed that in the normal population,(1) suggesting a unique relationship between these two uncommon entities. We report the case of a young child with Down syndrome and achalasia who was treated successfully with surgery.

\section{CASE REPORT}

The patient was born at term, with a birth weight of $2.64 \mathrm{~kg}$. She was noted at birth to have clinical features suggestive of Down syndrome, with subsequent genetic testing confirming the $47, X X,+21$ karyotype (nondisjunction). Other medical issues included congenital hypothyroidism, spontaneously closed patent ductus arteriosus with residual tiny patent foramen ovale, right pelviectasis that resolved spontaneously, and imperforate anus for which she underwent colostomy on the first day of life and a subsequent posterior sagittal anorectoplasty at four months of age.

The patient first had difficulty in feeding at 11 months of age when her parents reported one episode of choking. By the age of 14 months, she was having recurrent episodes of choking, particularly with solid feeds. Since the age of seven months, her weight had plateaued at around $7 \mathrm{~kg}$, dropping from the 75th to 25th percentile. Gastro-oesophageal reflux disease (GORD) was initially suspected and she was started on anti-reflux milk formula as well as omeprazole, ranitidine and domperidone. However, her symptoms persisted.

The patient was referred to a paediatric gastroenterologist when she was around 16 months old. At that time, she was vomiting half of her milk volume after almost every feed and was very symptomatic. A barium swallow study (Fig. 1) showed oesophageal dilatation with hold-up of contrast and tapering at the oesophago-gastric junction (i.e. the classical bird's beak or rat-tail appearance). Upper endoscopy showed retained food debris in the dilated distal oesophagus as well as poor distension of the oesophago-gastric junction with air insufflation. However, the child's parents declined oesophageal manometry, as they were not keen on further investigations but preferred to proceed with early definitive therapy. The patient eventually underwent Heller myotomy with Nissen fundoplication. Postoperatively, her vomiting completely resolved and she was able to tolerate both solids and liquids, resulting in good weight gain.

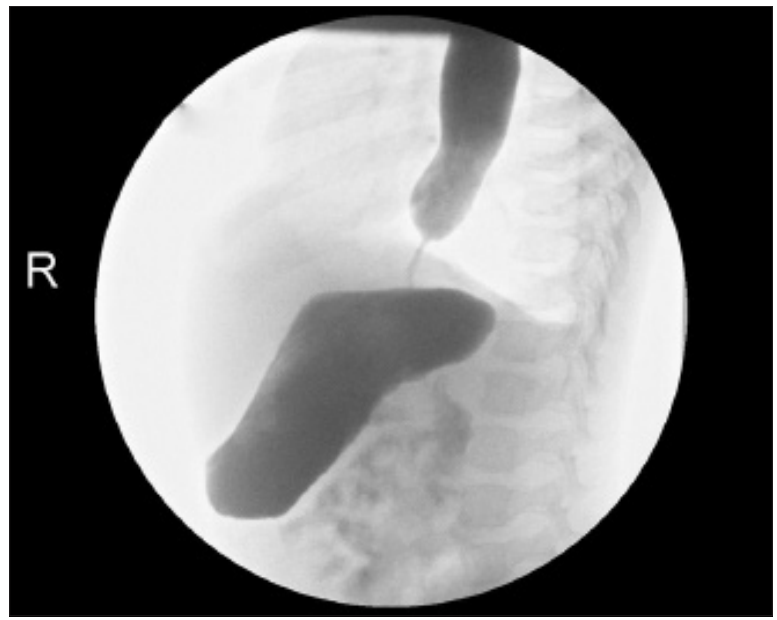

Fig. 1 Fluoroscopy image shows distal oesophageal dilatation and a classic bird's beak appearance on barium swallow. 


\section{DISCUSSION}

Achalasia is an uncommon condition, with an estimated prevalence of 8 per 100,000 people $\mathrm{e}^{(2)}$ and less than $5 \%$ of patients presenting before the age of 15 years. ${ }^{(3)}$ However, achalasia seems to be more frequent in patients with Down syndrome. For instance, Zárate et al reported two (3.4\%) patients with achalasia in a group of 58 children and adults with Down syndrome who were evaluated for oesophageal motor dysfunction. ${ }^{(1)}$

Although the exact aetiology of achalasia is unknown, studies have proposed that its pathogenesis may be related to autoimmune, infectious or genetic factors, ${ }^{(4,5)}$ leading to the intrinsic loss of inhibitory myenteric neurons in both the oesophagus and the lower oesophageal sphincter (LOS). The genetic imbalance in Down syndrome is thought to result in abnormal development of the nervous system, including the enteric nervous system, which may be due to decreased neuronal migration or abnormal dendritic development. ${ }^{(4}$ The aberrant immune response in Down syndrome, which is responsible for the increased risk of infection, may also be a contributing factor to the development of achalasia, as certain viruses such as varicella-zoster and measles have previously been implicated in the aetiology of achalasia. ${ }^{(5)}$ Another mechanism postulated for the pathogenesis of achalasia in Down syndrome is autoimmunity, which is highly associated with class II antigens of the major histocompatibility complex $(\mathrm{MHC})$ that are seen in both conditions. According to Zárate et al, the expression of these MHC class II molecules on oesophageal ganglion cells, when induced by interferon- $\gamma$ in response to inflammatory processes, could be a cause of ganglion cell destruction. ${ }^{(5)}$

Patients with achalasia typically present with a history of dysphagia initially with solids and later liquids as the condition progresses. However, dysphagia can be subtle and is not the most prominent symptom seen in children with achalasia, who more commonly present with vomiting instead. ${ }^{(6)}$ Hence, they may often be mistaken to have GORD, as in the case of our patient, thus delaying correct diagnosis and appropriate treatment. It may be prudent to investigate more extensively using either barium studies or manometry when symptoms of GORD are not typical or do not improve with empirical treatment in a child with Down syndrome.

There is currently no cure for achalasia as activity cannot be restored to the denervated oesophagus. The goal of treatment is the reduction of LOS pressure. Pharmacological modalities include the use of nifedipine, isosorbide dinitrate and sildenafil. However, relevant experience in children is limited, and thus, this approach may not be a good long-term option. ${ }^{(6)}$ Intrasphincteric injections of botulinum toxin should be reserved for high-risk candidates alone because the median duration of response is only four months, long-term efficacy may be reduced due to the formation of neutralising antibodies, and future surgical options may be compromised due to resultant perioesophageal inflammation and fibrosis. ${ }^{(6)}$ The two main treatment modalities with good reported success rates in adult patients with achalasia are pneumatic dilatation $^{(6)}$ and oesophagomyotomy. ${ }^{(7,8)}$ The therapy of choice for children, however, is not well established and depends on the expertise available at the institution. Pneumatic dilatation is less invasive, and has been found to be effective in more than $50 \%$ of patients, although patient may require repeated dilatations, with a risk of perforation each time and higher rates of postoperative dysphagia (up to $30 \%$ ). ${ }^{(6)}$ On the other hand, oesophagomyotomy, though more costly and invasive, offers more complete and longer duration of relief from dysphagia, ${ }^{(7)}$ with a rate of postoperative dysphagia of $5.2 \%$ but a higher rate of GORD $(3.8 \%){ }^{(6)}$ It is also reportedly feasible to perform oesophagomyotomy using minimally invasive techniques. ${ }^{(8)}$ For our patient, surgery was chosen in view of her young age and failure to thrive. An antireflux procedure was also performed to reduce the chances of postoperative GORD, for which there was a higher risk due to her underlying Down syndrome.

This report highlights the importance of early diagnosis of oesophageal motor disorders in Down syndrome patients. As achalasia and GORD mainly present with vomiting in children, it is essential to establish the right diagnosis through proper history-taking and early investigative studies so as to prevent complications such as failure to thrive and respiratory symptoms.

\section{REFERENCES}

1. Zárate N, Mearin F, Hidalgo A, Malagelada JR. Prospective evaluation of esophageal motor dysfunction in Down's syndrome. Am J Gastroenterol 2001; 96:1718-24.

2. Wong RKH, Maydonovitch CL. Achalasia. In: Castell DO, ed. The esophagus. 1st ed. Boston: Little Brown, 1992: 233-60.

3. Mayberry JB, Mayell MJ. Epidemiological study of achalasia in children. Gut 1988; 29:90-3.

4. Moore SW. Down syndrome and the enteric nervous system. Pediatr Surg Int 2008; 24:873-83

5. Zárate N, Mearin F, Gil-Vernet JM, Camarasa F, Malagelada JR. Achalasia and Down's syndrome: coincidental association or something else? Am J Gastroenterol 1999; 94:1674-7.

6. Rosen R, Nurko S. Other motor disorders. In: Walker WA, Goulet $O$, Kleinman RE, Sherman PM, Shneider BL, Sanderson IR, eds. Pediatric Gastrointestinal Disease. Ontario: BC Decker, 2004: 424-62.

7. Morris-Stiff G, Khan R, Foster ME, Lari J. Long-term results of surgery for childhood achalasia. Ann R Coll Surg Engl 1997; 79:432-4.

8. Tannuri AC, Tannuri U, Velhote MC, Romão RL. Laparoscopic extended cardiomyotomy in children: an effective procedure for the treatment of esophageal achalasia. J Pediatr Surg 2010; 45:1463-6. 